\title{
1.000 Teilnehmer beim 3. DENTSPLY Implants Kongress
}

Richtig entscheiden - Patienten begeistern: Unter diesem Motto fand der 3. DENTSPLY Implants Kongress (DIKON) vom 18. bis 19. September 2015 in Berlin statt. Mehr als 1.000 Besucher waren in das Designhotel "andel's" gekommen, um in Vorträgen und Workshops ihre implantologischen Kenntnisse auf den neuesten Stand zu bringen. Eingerahmt wurde der Kongress von PEERS-Veranstaltungen, die sich dem Thema "Lebensqualität im Alter" und "Implantate im zahnmedizinischen Umfeld" widmeten. PEERS (Platform for Exchange of Experience, Education, Research and Science) ist ein von DENTSPLY Implants unterstütztes internationales Expertennetzwerk mit Mitgliedern aus Klinik, Praxis und Zahntechnik.

Fünosen ür eine kleine Anwendergruppe von OsseoSpeed Profile EV, dem neuen Implantat für den schräg atrophierten Kieferkamm, begann die Veranstaltung bereits am 17. September mit dem Pre-Kongress. Privatdozent Dr. Robert Nölken stellte den Teilnehmern das neue Profile-Implantat des ASTRA TECH Implant System EV und seine vielfältigen Anwendungsmöglichkeiten in der implantologischen Praxis vor. Die Kollegen diskutierten die Indikationen und Versorgungsmöglichkeiten mit dem innovativen Implantatsystem und brachten ihre eigenen Erfahrungen aus der Praxis ein.

Das große PEERS-Forum zu Beginn des DIKON stand im Zeichen der Alterszahnheilkunde und wurde von Prof. Dr. Hans-Christoph Lauer moderiert. Die Referentin Prof. Dr. Ina Nitschke berichtete über die Herausforderungen bei der Behandlung alter Menschen. Innovationen wie intraorale Scanner und ein kompletter digitaler Workflow können den Zahnarzt gerade bei der Therapie alter Menschen künftig wirkungsvoll unterstützen, was durch die Referenten Dr. Sebastian Quaas und Dr. Aiste Gintaute in ihren Vorträgen anschaulich belegt wurde. Große Aufmerksamkeit erregte bei den Teilnehmern der "AgeMan", ein Anzug, der aufgrund seines Gewichtes und Gestaltung die Einschränkungen im höheren Alter simuliert und seinem Träger ein Gefühl dafür vermittelt, welche Funktionen im Alter eingeschränkt sind. Prof. Dr. Meike Stiesch referierte zum Abschluss des Vormittags über Prävention und Therapie der Biofilmbildung auf implantatgetragenem Zahnersatz bei älteren Patienten.

Im ersten Vortragsblock des Kongresses am Freitagnachmittag unter der Moderation von Prof. Dr. Dr. Knut Grötz beschäftigten sich die Referenten mit Innovationen für die Praxis. Dr. Mischa Krebs zeigte den aktuellen Stand zum digitalen Workflow in der Implantologie auf. Mit den Planungs- und Fertigungskomponenten SIMPLANT und ATLANTIS sind Sofortversorgungen im kompletten digitalen Workflow möglich, wobei zum Zeitpunkt der Implantation schon die präfabrizierten individuellen Abutments und Provisorien bzw. definitiven Kronen vorliegen. Dr. Peter Gehrke sprach über die Oberflächengüte von CAD/CAM-gefertigten Abutments, deren Oberflächenrauigkeit etwas größer ist als bei Standardabutments. Diese Rauigkeiten differieren je nach verwendetem System erheblich. Darüber hinaus zeigte er einen effizienten Reinigungsprozess, um Verunreinigungen an der Oberfläche vor dem Einsetzen des Abutments wirkungsvoll zu entfernen. Die komplexe Therapie groBer Defektsituationen nach Tumorentfernung und Unfällen stellte Prof. Dr. Dr. Jürgen Hoffmann anhand zahlreicher Fälle eindrucksvoll vor. Anschließend präsentierte Privatdozent Dr. Robert Nölken zusammen mit Dr. Dr. Eik Schiegnitz die aktuellen Ergebnisse der Weichgewebsentwicklung an Implantaten im Rahmen der German ASTRA Soft Tissue Study (GAST), die an der Universität in Mainz durchgeführt wird.

Zahlreiche Workshops wurden angeboten, um implantologisches Wissen gezielt vertiefen zu können. Diese behandelten die Schwerpunkte
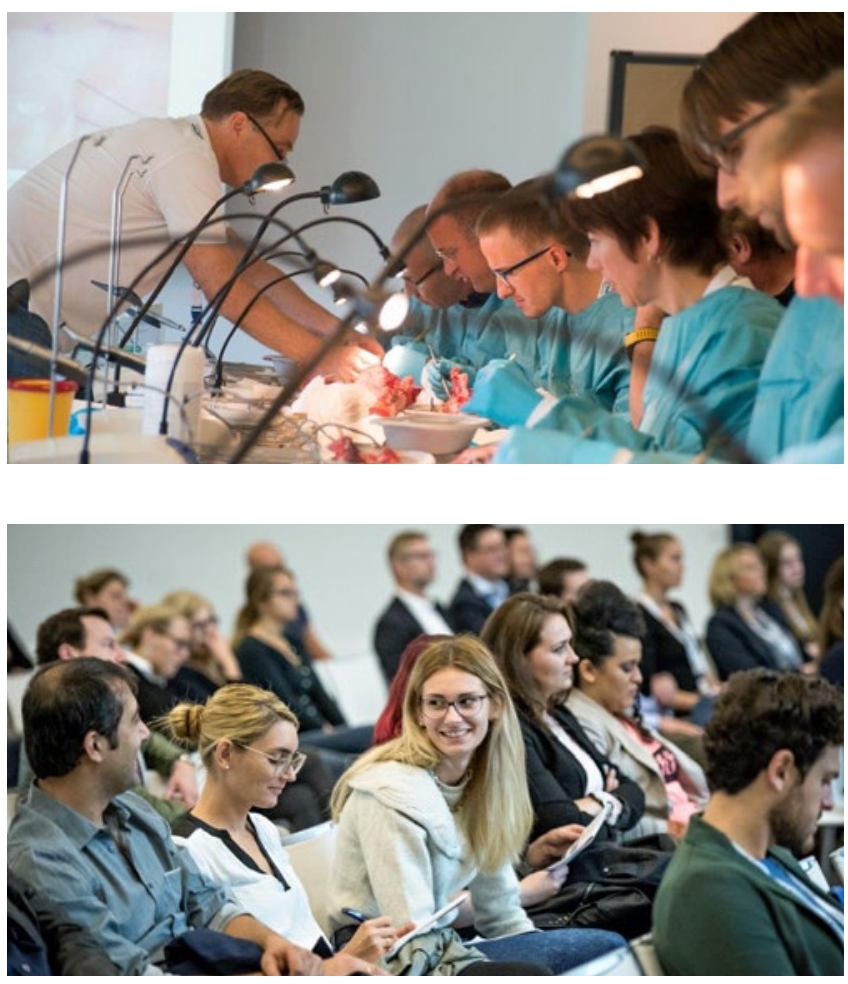

Digitale Zahnmedizin, Hart- und Weichgewebsmanagement und Versorgungskonzepte. Aber auch Themen zum Praxismarketing und Praxismanagement wurden in Workshops abgehalten. Dr. Karsten Wagner, Geschäftsführer DENTSPLY Implants Deutschland, bezeichnete die Workshops in der Pressekonferenz zum Auftakt des DIKON als „Herzstück der Veranstaltung“, das zu einem modernen Kongress dazugehört: „Ich bin fest überzeugt, dass diejenigen Teilnehmer, die hier die Möglichkeit haben, selbst Hand anzulegen, viel für die tägliche Arbeit und die tägliche Praxis mitnehmen."

Im Rahmen der Abendveranstaltung am Freitag fand die feierliche Verleihung der PEERS-Förderpreise 2015 für Nachwuchswissenschaftler statt. Ausgezeichnet wurden Arbeiten in verschiedenen Kategorien - Dr. Eleftherios Grizas und ZTM Ulrich Hauschild erhielten die Preise für die besten Fallpräsentationen, ZTM Daniel Seebald gewann den „Implantat-Prothetik-Preis", Dr. Paula Korn wurde zur Preisträgerin der besten Publikation gekürt und Privatdozent Dr. Dr. Daniel Rothamel erhielt den Preis für das beste Poster.

Weitere Informationen unter www.dentsplyimplants.de 